\title{
THEORY OF STELLAR CORONAE
}

\author{
S. Serio \\ Astronomical Observatory and IAIF-CNR \\ Palazzo dei Normanni \\ 90134 Palermo, Italy
}

\begin{abstract}
X-ray observations have shown in recent years that the existence of high temperature plasmas in stellar atmospheres is far more widespread than extrapolated from preexisting theories of the solar and stellar coronae, forcing a radical change in our understanding of the mechanisms of coronal physics. This paper reviews our current ideas on stellar coronae, in particular on the role of magnetic confinement in the atmospheres of late type stars.
\end{abstract}

\section{INTRODUCTION}

The new field of stellar coronal physics enjoys already of a large and consistent set of observations (Vaiana and Sciortino 1987). Moreover, much of our understanding of the theoretical aspects of stellar coronae relies on the high level of detail and high spatial resolution of solar coronal observations. Since the extension of solar coronal theory to stellar coronae requires its extrapolation over a wide range of parameters, the comparison of theory and observations for stellar coronae provides also a feedback on our understanding of the solar corona.

The high degree of spatial structuring is among the most striking characteristics of the solar corona. It is easily interpreted as a manifestation of the existence of coronal magnetic fields, that shape the low $\beta$ coronal plasma (Vaiana and Rosner 1978). The general idea is, therefore, that stars similar to the Sun, i. e. stars in which convective motions can interact with rotation to generate magnetic fields, can experience similar phenomena. This picture assumes that the magnetic fields produced by a dynamo mechanism inside the stars cause coronal and chromospheric activity, and feedback, through mass loss and magnetic spindown, on the rotation rate and differential rotation that are, together with convection, the essential ingredients of the dynamo. High energy particles produced during flares, and loss of mass through the wind, create a link of coronal physies to the physics of the circumstellar medium.

Since the picture is complicated by the feedback loops, and by the richness of the phenomena in magnetized plasmas, it is easily understandable that many problems are still open. In some of the basic areas of coronal physics, such as those relating to the heating of the coronal atmosphere, and to the source of the magnetic field, the theory does only provide a qualitative framework to understand the basic phenomena, rather than detailed predictions.

Although one might think of a role for some primordial magnetic fields (see for example Uchida, 1986) in early type stars, the picture that has attracted some consensus for the coronae of these stars, is one in which high temperatures plasmas are produced by instabilities and shocks in their strong winds (Lucy and White 1980; Lucy 1982). 
Since the theory of these instabilitis is treated elsewhere in this volume (Hearn 1987), I ball limit my discussion only to late type coronae. In the progress of this paper I shall review some of the critical aspects of the theory of magnetically confined coronae, namely the dynamo mechanism and the formation and heating of the coronal structures (\$ 2). I shall then concentrate on "loop models" of stellar coronae of late type stars. This last topic has been recently investigated by several authors (Zolcinski et al. 1982; Giampapa et al. 1985; Schmitt et al. 1985; Landini et al. 1985; Stern et al. 1986; Mewe 1986), and it constitutes, perhaps, the area of coronal physics most satisfactory for comparison of theory and observations.

\section{CORONAE OF LATE TYPE STARS}

Our understanding of the coronal mechanism in late type stars is somewhat guided by observations of the solar corona showing that it is dominated by magnetic fields; that these fields are highly structured forming what we call loops or complexes of loops; that the coronal plasma is highly dynamic, with characteristic times ranging from the few minutes of flares to the several months of long term evolution of active regions.

The questions that $I$ will address in this context include: 1 . the production of magnetic fields, 2 the heating of the magnetized plasma, and 3 . the structuring of the magnetized plasma.

\subsection{Production of magnetic fields - Dynamo}

Magnetic fields in late type stars are supposed to be produced by a dynamo mechanism. To understand how this mechanism works, let's look at the diffusion equation for the magnetic field II:

$$
\frac{\partial \underline{H}}{\partial t}=\underline{\nabla} \times(\underline{v} \times \underline{H})-\nabla \times(\eta \underline{\nabla} \times \underline{H}),
$$

where $\underline{v}$ is the fluid velocity and $\boldsymbol{\eta}$ is the diffusivity.

Owing to the presence of velocity eddies, this equation is intractable. However, if one averages over eddies, one gets the equation for the mean field $\underline{B}$ :

$$
\frac{\partial \underline{B}}{\partial t}=\underline{\nabla} \times[(\underline{\Omega} \times \underline{r}) \times \underline{B}+\alpha(r) \underline{B}]-\underline{\nabla} \times\left(\eta_{t} \underline{\nabla} \times \underline{B}\right),
$$

where $\Omega$ is the rotation speed. The " $\alpha$ " term and the " $\Omega$ " term drive the dynamo, while the $\eta$ (turbulent diffusivity) term acts as a damping term. If we now neglect this last term and assume that $\underline{B}$ consists of a main poloidal component derived by a vector potential $A$ in the azimuthal direction, and by a seed azimuthal component $B_{\phi}$, we find:

$$
\frac{\partial^{2} B}{\partial t^{2}}=r\left[\underline{\nabla}\left(\alpha B \phi_{\phi}\right) \times \underline{\nabla}(\Omega \sin \theta)\right]+0\left(\alpha^{2}\right) \text {. }
$$

Therefore, we can easily see that $B_{\phi}$ grows with a rate

$$
\tau^{-1} \approx \sqrt{\alpha|\underline{\nabla} \boldsymbol{\Omega}|}
$$

Obviously, for a strong amplification we expect that the newly created $B_{\phi}$ stays around at least for a time $\sim \boldsymbol{\tau}$.

It was pointed out by Parker (1975), that a magnetic flux tube embedded in the convection zone experiences a buoyancy force, that lifts it to the surface with a rate of the order of the 
Alfvèn speed. For exampie, if the flux tube is created at the depth of $\sim 10^{10} \mathrm{~cm}$, the time of rise is of the order of one day - too short for appreciable amplification - and field amplification has to take place in a region where the field can be stored long enough. This region can be identified in the overshoot layer at the top of the radiative zone. There, the drag force caused by downward flows is not balanced by buoyancy, and the result is that flux tubes can leave enough for magnetic amplification.

As suggested by Schmitt and Rosner (1983), this configuration is eventually unstable. Vertical motions of thin flux tubes will tend to be amplified because of the difference in magnetic and thermal diffusivity (doubly diffusive instability).

The time scale of the instability can be estimated by simply taking the pressure scale height at the overshoot layer $L$, divided by the Alf vèn velocity. This results in the constraint $\tau \sum L / v A$ A rough estimate of $\alpha$ in Eq. 2 can be given by $\alpha \propto L / R / R$, where $R$ is the stellar radius, implying:

$$
B \sim \sqrt{L^{2}(\Omega|\underline{\nabla} \Omega| \rho / R)},
$$

where $\rho$ is the mass density.

Since simple models for magnetic heating of coronae predict that the power going into heat is $\propto B^{2}$ (e. g. Golub et al. 1980), a quadratic relationship among $X$-ray luminosity and angular velocity is predicted, in general accord with observations (Pallavicini et al. 1981a), whenever E $\Omega$ scales as $\boldsymbol{\Omega} / \boldsymbol{R}$.

For early $F$ type stars, however, the dependence on $\mathbf{Q}$ is masked by the strong dependence of the depth of the convection zone (or of the convective turnover time) on mass; hence one expects, as found in the survey of Schmitt et al. (1985), that the X-ray luminosity of the early F stars be correlated to Rossby number rather than to angular velocity. Moreover, when the gradient of angular velocity is not fully developed, simple correlations are lost. Example: the Pleiades $G$ and $K$ stars, which show little correlation between $X$-ray luminosity and rotational velocity, but also between X-ray luminosity and Rossby number (Micela et al. 1984, 1985).

In summary, dynamo theory is in rough agreement with observations, although a detailed comparison of theory and observations is not yet possible because of the complicated physics of convection in the magnetized and rotating stellar interior.

\subsection{Structuring and Heating of the stellar coronae.}

The next problem I want to address is that of structuring and heating of the coronae. The question is: how does the magnetic field emerging at the photosphere acquire the fine structuring in thin elongated loops?

One approach has been pursued by Ferrari, Rosner, and Vaiana et al. (1982) and by Bodo et al. (1985). They consider a vertical magnetic field embedded in the cool photosphere, with a current parallel to the field produced by photospheric motions. they show that such configuration is unstable against Joule heating. Increased local heating increases the current locally, in a runaway process, thus producing filamentation on a small scale.

Of course this result pertains only to the linear regime, but it gives, nonetheless, an indication that the coronal structures are highly filamented. This might help to solve an other problem, that about heating the corona. The essential starting point, and the difficulty of the topic, stays in the vastness of the 800 of instabilities in magnetized plasma that can contribute to the thermal balance of the corona. The basic picture is that of a loop anchored in the photosphere. Turbulent motions of the footpoints will induce both MHD waves propagating along the field, and currents parallel to the field. The dissipation of the waves (AC heating) and the possibly anomalous Joule heating by the currents (DC heating) both contribute to coronal energy balance (Kuperus, Ionson, and Spicer 1981; Serio 1983). 
To determine which of the two mechanism is most important, and its detailed working, it is extremely difficult (if not impossible because of concurrence of different mechanisms). DC heating has generally the shortcoming that narrow current sheets are necessary, thus rising the problem of heat transport across the field lines. AC heating models using body waves or Alfvèn waves do not encounter this problem, but the heating rates are difficult to estimate.

As for current heating, it has been suggested that observations of microwave emission from dwarf $M$ flare stars can be explained in terms of coronal currents (Holman 1986). The evidence comes because the microwave emission observed during flares in dMe stars can be attributed to gyrosyncrotron emission from high energy ( $10 \mathrm{keV}$ ) electrons (Linkky and Gary 1983). On the other side, an important feature of current dissipation mechanisms is that, in a plasma at temperature $T$, in the presence of an electric field $E$, thermal electrons with velocity greater than the critical velocity defined by $v_{c}^{2}=\left[m v_{e}(K T / m)^{3 / 2}\right] / e E$, where $\nu_{e}$ is the thermal collision frequency, will be accelerated freely. Holman $(1985,1986)$, finds that a current dissipation model with current drift speed of the order of the ion sound speed can accelerate enough electrons to explain both $\mathrm{X}$-ray and microwave gyrosyncrotron emission in flares and in the stationary emission of dMe stars.

\section{CORONAL LOOP MODELS}

The effect of confinement by the magnetic field on a static coronal plasma is to force scaling relationships for base pressure $p$, maximum temperature in the loop $T$, loop length $L$, and volumetric heating rate $Q$ (Rosner, Tucker, and Vaiana 1978).

$$
\begin{aligned}
& T \sim 1.4 \times 10^{3}(p L)^{1 / 3} \mathrm{~K}, \\
& Q \sim 10^{5} p^{5 / 6} L^{-7 / 6} \mathrm{ergs} \mathrm{cm}^{-3} s^{-1} .
\end{aligned}
$$

Although individual loops may have a wide range of base pressures and length, those contributing most to $X$-ray emission for unresolved stellar observations, will have a length about equal to $1 / 2$ the pressure scale height $h$. The emission measure above that height, in fact, will be negiigible, while smaller loops are likely to fill a small fraction of the available coronal volume. Under these assumptions, it is easy to see that the $\mathrm{X}$-ray luminosity is related to coronal temperature by:

$$
L_{x} \sim 5.5 \cdot 10^{11} T^{5 / 2} q(h / R) f \operatorname{ergs~}^{-1} \text {, }
$$

where $q(h / R)$ is a slowly varying function of the ratio of the pressure scale height to the stellar radius $R$, and $f$ is a "filling factor", describing the fraction of photospheric surface covered by the footpoints of coronal loops (Rosner, Golub, and Vaiana 1983; Stern et al. 1981). Eq. 8, with $f=1$, sets an upper limit on the X-ray luminosity of a stellar corona. Alternatively, it can be used, once the X-ray luminosity and coronal temperature are known, to estimate the surface filling factor.

The question naturally arising is whether our picture, i.e. that of a corona dominated by identical static loops of "convenient" height, is realistic or not. This question cannot be answered unless we have an independent way of estimating $f$ in Eq. 8, or unless we use some more refined means, such as, for example, comparing the distribution of emission measure predicted by coronal models with that deduced from observations. Static and dynamic loop models shall be discussed in the following subsections.

\subsection{Static Loop Models}

Assuming uniform density and temperature across the loop's cross section, the model involves the (numerical) solution of the unidimensional energy and force equations for a plasma confined in a 
tube of assigned shape and dimensions,:

$$
\begin{aligned}
& Q=n^{2} P(T)-\frac{\partial F_{c}}{\partial s}, \\
& n \mu_{B_{s}}=\frac{\partial p}{\partial s},
\end{aligned}
$$

where $n$ is the plasma density, $P(T)$ is the plasma emissivity (emitted power per unit emission measure), $F_{c}$ is the conductive flux, $\mu$ is the average ion mass, and $g_{s}$ is the component of gravity along the loop's coordinate $s$. The boundary conditions usually used for this model assume that the loop is symmetric at the top, and that the conductive flux $F_{c}$ vanishes at the footpoints (e.g. Rosner, Tucker, and Vaiana 1978), although different conditions have also been discussed (e.g. Vesecky, Antiochos, and Underwood 1979).

Hydrostatic numerical models of the kind described in Eqs. 9 and 10 have been developed for the solar corona (Vesecky, Antiochos and Underwood 1979; Serio et al. 1981) and have been shown to be generaily in good agreement with resolved observations of active region loop complexes (Pallavicini et al. 1981b).

The extrapolation to stellar coronae, however, is not straightforward: owing to the lack of epatial resolution, it involves the assumption, as discussed above, that only loops of one size and base pressure are important in determining the observational characteristics of the corona. Despite this unavoidable strong assumption, static models have been applied to a variety of stellar coronal observations (Giampapa et al. 1985; Schmitt et al. 1985; Landini et al. 1985; Stern et al. 1986). Two different methods have been used, one relying on the comparison of the predicted emission in the EUV region and in the X-ray region (Giampapa et al. 1985; Schmitt et al. 1985; Landini et al. 1985) with IUE and Einstein or Exoset observations, the other on the detailed fit of the emission predicted by the model and the observed pulse height spectral distribution in the Einstein Imaging Proportional Counter (IPC - Schmitt et al. 1985; Stern ot al. 1986).

To illustrate the relative merits of both approaches, and their fundamental limitations, I shall briefly review some of their applications. Testing the basic assumption, i.e. that a corona can be characterized by a single loop, is particularly desiderable to establish the validity of the approach. This can be done on the only star for which we have resolved X-ray and EUV observations: the Sun. Giampapa et al. (1985), have used full disc estimates of EUV and X-ray solar fluxes to constrain single static loop models of the solar corona both during solar maximum and solar minimum.

The procedure for the fitting is straightforward. One builds a series of static models having the maximum temperature suggested by the $X$-ray observations, but differing in base pressure (the constraint of confinement and the fixed maximum temperature do obviously limit the parameter space to a trajectory in the $p L$ plane -- Eq. 6). For each model one computes the predicted fluxes, assuming that the emission comes entirely from identical loops whose integrated footpoint area is a fraction of the stellar surface:

$$
F_{i}=f_{i} \int^{2} C_{i}(T) d s \text {. }
$$

where $F_{i}$ is the line flux in any of the EUV lines under consideration (whose line emissivity function is $C_{i}(T)$ ), or the $X$-ray flux (here $C_{i}(T)$ is the X-ray emissivity function folded through the instrument bandpass). If the rhs of Eq. 11 is evaluated for $n$ different EUV lines and/or X-ray bandpasses, and compared to the corresponding observed fluxes, it gives $n$ values of $f_{i}$, whose mean represents the "filling factor" for the model, and whose scatter is a measure of how good, or how bad, is the loop model we are testing. In this way it is generally possible to select the loop model for which the scatter of the different $f_{i}^{\prime} s$ is minimum; the length of the 
model loop and the average filling factor will then be a representation of the data. In the Sun this can be compared to direct, spatially resolved observations, to assess the validity of the approach. The "best fit" models for solar minimum and solar maximum have loop lengths 7.0-10 10 and $6.9-10^{9} \mathrm{~cm}$, and filling factors 0.56 and 0.03 . While deviation of individual filling factors are appreciable, this appears to be a fair representation of the coronal structures in both cases: large scale structures dominating most of the corona during solar minimum, and active regions covering a small fraction of the solar surface during solar maximum.

Although this approach appears to work fairly well for the Sun, one should be careful in interpreting its results for other systems, for at least the following reasons:

i)

EUV and X-ray data are usually not simultaneous, thus we have to rely on the additional hypothesis that no significant variations have occurred between the times of acquisition of the different sets of data;

ii)

the actual heating distribution along the loop is difficult to assess, while in the discussion above it has been assumed uniform; a different distribution makes little difference for the X-ray flux, but it may give significantly different values for the EUV fluxes;

iii) single loop models, while realistic for the solar corona, may not be realistic in the wide stellar context (e.g. Jordan et al. 1986).

In the second approach, only the X-ray spectral information is used. Here one computes the deviations of the IPC pulse height spectra from the predictions of the model. One may think that, having more degrees of freedom (the number of IPC channels available for the fit is generally 210 ), one can break the "degeneracy" implied in Eq. 6 , and find a minimum $\chi^{2}$ in the $p L$ plane. This is not true in general (Serio 1985; Schmitt et al. 1985), but by using more parameters, such as, for example, a variable cross section of the loops, one can obtain some constraints on the model parameters from the data (Stern et al. 1986). However, since the surfaces of constant $\chi^{2}$ have no sharp minima in the parameter space, the probable error in the determination of the parameters is inherently high. Thus, even in this case, loop models suffer some inadequacy, and we may think of their validity in a statistical rather than individual way, in the sense that the ensemble of a large number of best fit loop models is representative of the ensemble of stellar loop atmospheres. The problem, at this stage in the analysis of the Einstein data, is that we do have only a few fitted models, although we might have more in the near future.

\subsection{Dynamic Loop Models.}

The success of hydrodynamic loop models in describing the evolution of solar flares (Strong 1986; Peres et al. 1987) is certainly stimulating and pointing to the possibility that similar models can be applied to stellar flares. These models are based on the equations of mass, momentum, and energy conservation, and on the equations of state and of ionization balance for plasma confined in a rigid tube and subject both to steady-state and transient heating:

$$
\begin{aligned}
& \frac{d n}{d t}=-n \frac{\partial v}{\partial s}, \\
& n \mu \frac{d v}{d t}=-\frac{\partial p}{\partial s}+n \mu_{g_{s}}+\frac{\partial}{\partial s}\left\{\lambda \frac{\partial v}{\partial s}\right\}, \\
& \frac{d E}{d t}+(E+p) \frac{\partial v}{\partial s}=0-n^{2} \beta P(T)+\lambda\left(\frac{\partial v}{\partial s}\right)^{2}+\frac{\partial F_{c}}{\partial s},
\end{aligned}
$$




$$
\begin{aligned}
& p=(1+\beta) n K T, \\
& E=\frac{3}{2} p+n \beta X,
\end{aligned}
$$

where $\checkmark$ is the plasma velocity, $\lambda$ its viscosity coefficient, $Q$ describes both the steady state and the transient volumetric power input, $\beta$ is the ionization fraction, and $\boldsymbol{X}$ the hydrogen ionization potential.

Peres of al. (1983) have shown that the static solutions of Eq. 10 and 11 are indeed stable under the dynamics described by Eqs. 12-16, if one takes into account also the response of the chromosphere to coronal transients, i.e. if the modeling is extended to the denser layers below the transition region, that can act as a plasma reservoir.

Fig. 1 shows the comparison of X-ray line fluxes derived by numerical solutions of Eqs. 1216. and of X-ray observations of a compact solar flare (Peres et al. 1987).

Fig.1 Comparison of computed (solid lines) light curves of X-ray lines, and of observations by the SMM Flat Crystal Spectrometer for the solar flare of 1980, Nov. 12, 17:00 UT. The computations assume that the flare impulsive energy is deposited near the top of a $100 p 2 \cdot 10^{9} \mathrm{~cm}$ high. The time profile of the impulsive heating, as well as its peak value, are optimized to best fit the observations. The flux is in units of photons $\mathrm{cm}^{-2} \mathrm{~s}^{-1}$ at Earth (Peres et al. 1987).
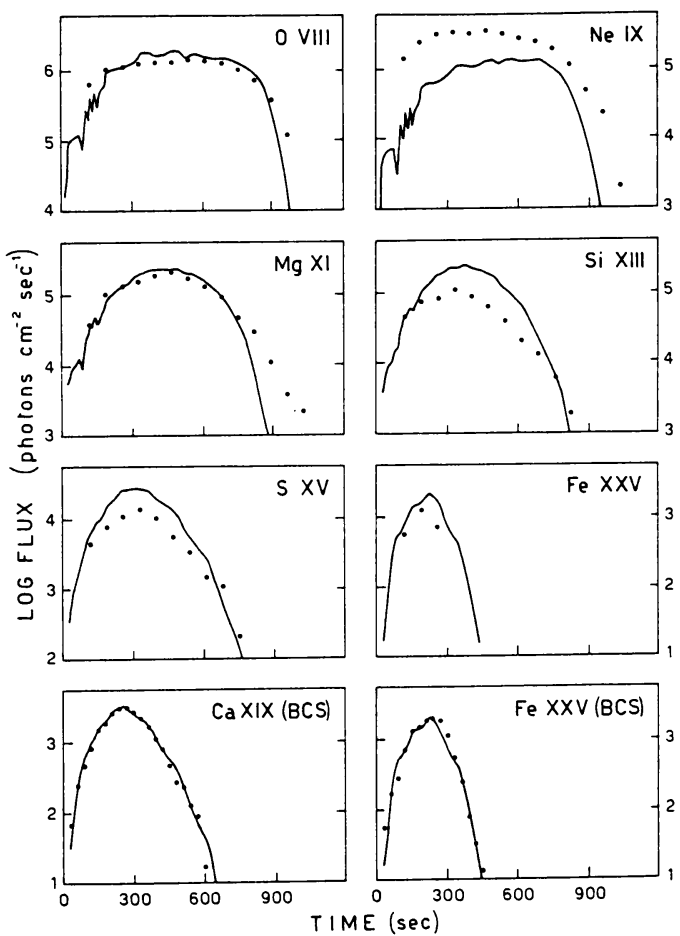

It is apparent that the evolution of the flare is well described. Of course, for a solar flare observed with some spatial resolution, the dimensions of the loop in which the flare is supposedly exploding are known, and the numerical calculations, therefore, can be used to get some insight into the physics of the heating mechanism phenomenon, for example the site of energy release.

For stellar flares, however, the basic information we can hope to extract from a dynamic model is the very same spatial picture of the flaring region that we cannot perceive directly. Since the dynamics of plasma cooling is sensitive to the dimensions and shape of the region in which it is confined, we can hope to be able to obtain some information on the structure of stellar coronae just by comparing numerical calculations under different hypothesis with observed light curves of $X$-ray stellar flares. To do this in a simple way, we have to assume that the 
flare is confined during its evolution, which inevitably limits our considerations to small flares.

The approach is therefore the following: estimate the total impulsive energy related to the flare and the duration of the impulsive phase; assume starting values for the length and the cross section of the model loop, from the observed decay rate and of the integrated count rate, and compute the evolution of the plasma it confines, subject to the corresponding transient energy input; finally, compare the cooling rate of the computed light curve with the observed one, to estimate how the length of the loop, in a successive iteration, has to be changed to provide a better agreement.

In this way it is possible, in principle, to determine the best parameters of the loop (length and cross section) corresponding to a given pattern of impulsive energy input; successive refinements can be obtained by acting on the time evolution of the impulsive energy term and/or its total amount, and comparing computed with observed spectra (IPC spectra, for example, for observations with Einstein).

This approach is being followed by Reale et al. (1987) in a study of one flare observed by Einstein on Prox Cen. Some preliminary results of this study are illustrated in Fig. 2, showing the sensitivity of the flare cooling rate to the length of the model loop, and in Fig. 3, which compares the evolution of the computed average loop temperature with that measured by a isothermal fit to the IPC data.

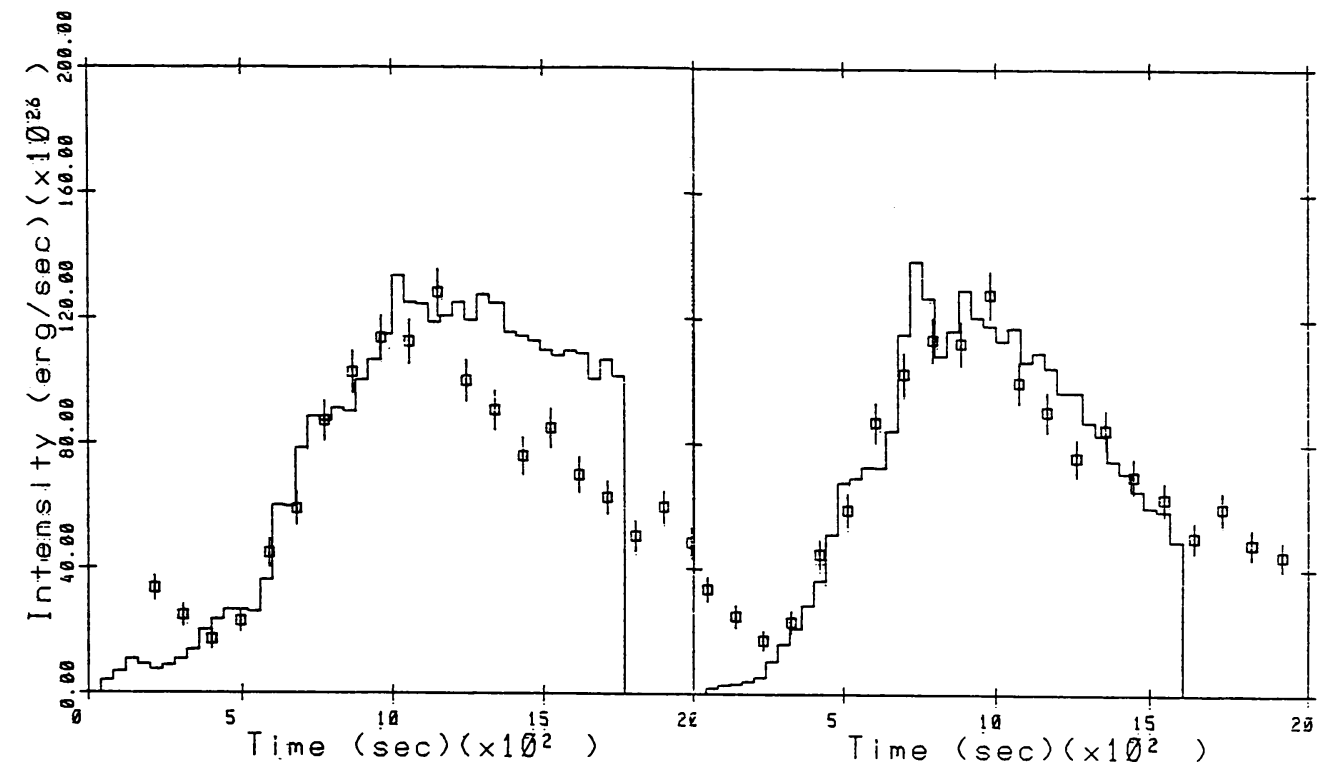

Fig.2 Comparison of computed (solid lines) $\mathrm{X}$-ray light curves and Einstein $\mathrm{WC}$ observations for a Prox. Cen. flare: a) in a loop $1.10^{10} \mathrm{~cm}$ high; b) in a loop $5.10^{5}$ high, with the came energy input as in a).

As for the solar flare, the agreement between calculations and observations is good, and this is certainly comforting, considering the large amount of computing time that is necessary to run the numerical code. A few words of caution are of order, however. Some of the basic hypothesis underlying the simple physics described by Eq. 12-16 are difficult to verify for a 
Fig.3 Comparison of the average nlasma temperature cierived by the hydrodynamic model for the Prox. Cen. flare and IPC single temperature fits to the observed count distribution.

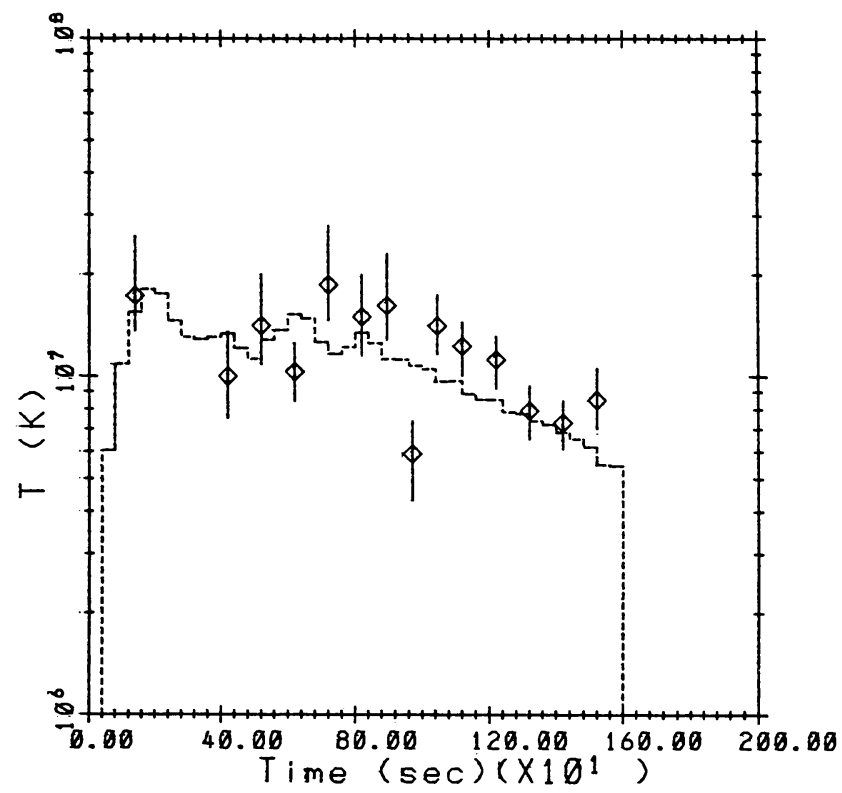

stellar flare (for example that of confinement throughout the evolution), and therefore the agreement between calculations and observations can hardly be construed as a proof of the validity of the loop model itself, but rather as an independent confirmation of the wide body of evidence, from the solar analogy to the success of static loop models, pointing to a picture of stellar coronae as dominated by magnetic structures.

\section{SUMMARY AND CONCLUSIONS}

Although stellar coronae have been observed only recently, we have already a wide body of data and a consistent framework for their theoretical understanding, especially for late type stars where the Solar analogy can be used.

According to our picture, the coronae of late type stars, similarly to the solar corona, are confined and heated by the magnetic fields that also shape the coronal plasma in loop-like structures. Models trying to explain the observations by the emission of plasma confined in a static magnetic loop are reasonably successful, although they are not entirely constraining the physical parameters of the loop atmospheres, because of the high conductivity of the coronal plasma and of the poor spectral resolution of available observational data. Moreover, the assumption on which they are based, that the coronal emission is dominated by loops of the same size and base pressure is difficult to test, although for the Sun it vorks fairly well. Models describing the dynamic evolution of plasma flaring in a compact loop are more promising in this respect, because the flare event is presumably occurring in a localized coronal region whose emission can easily overwhelm the background coronal emission.

More theoretical insight and a post Einstein generation of stellar X-ray observations, however, will have to address several problems relating to stellar physics, including the origin of $X$ ray emission in early type stars, the dependence of late type stellar X-ray emission on parameters such as age, rotation rate, and the depth of the convection zone, the determination of the boundary of the region of the HR diagram in which dynamo activity is present, the contribution of flares and winds to the mass budget of the interstellar medinm and to the cosmic ray flux, and a more detailed picture of the geometrical and thermal structure of late type coronae. 


\section{ACKNOWLEDGMENTS}

This work was supported by Piano Spaziale Nazionale and Ministero Pubblica Istruzione.

\section{BIBLIOGRAPHY}

G. Bodo, A. Ferrari, S. Massaglia, R. Rosner, and G. S. Vaiana, 1985, Ap. J., 291, 798.

A. Ferrari, R. Rosner, G. S. Vaiana, 1982, Ap. J., 263, 944.

M. Giampapa, L. Golub, G. Peres, S. Serio, and G. S. Vaiana, 1985, Ap. J., 289, 203.

L. Golub, C. Maxson, R. Rosner, S. Serio, and G. S. Vaiana, 1980, Ap. J., 238, 343.

A. G. Hearn, 1987, this volume.

G. D. Holman, 1985, Ap. J, 293, 584.

G. D. Holman, 1986, preprint.

C. Jordan, A. Brown, F. M. Walter, and J. L. Linsky, 1986, MNRAS, 218, 465.

M. Kuperus, J. A. Ionson, and D. S. Spicer, 1981, Ann. Rev. Astron. Ap., 19, 7.

M. Landini, B. C. Monsignori Fossi, F. Paresce, and R. A. Stern, 1985, Ap. J., 289, 709.

J. L. Linsky, and D. E. Gary, 1983, Ap. J., 274, 776.

L. B. Lucy, 1982, Ap. J., 255, 286.

L. B. Lucy, and R. L. White, 1980, Ap. J., 241, 300.

R. Mewe, 1986, Proc. of the COSPAR Symposium on Stellar and Solar Activity (Toulouse), in press.

G. Micela, S. Sciortino, and S. Serio, 1984, in M. Oda and R. Giacconi (eds.): X-Ray Astronomy '84, Tokyo (Inst. of Sp. and Astronau. Sci.), 43.

G. Micela, S. Sriortino, S. Serio, G. S. Vaiana, J. Bookbinder, F. R. Harnden Jr., L. Golnb, and R. Rosner, 1985, Ap. J., 292, 172.

R. Pallavicini, L. Golub, R. Rosner, G. S. Vaiana, T. Ayres, and J. L. Linsky, 1981a, Ap. J., 248, 279.

R. Pallavicini, G. Peres, R. Rosner, S. Serio, G. S. Vaiana, 1981b, Ap. J., 247, 692.

E. N. Parker 1975, Ap. J., 198, 205.

G. Peres, F. Reale, S. Serio, and R. Pallavicini, 1987, Ap. J, in press.

G. Peres, R. Rosner, S. Serio, and G. S. Vaiana, 1983, Ap. J., 252, 791.

F. Reale, et al., 1987, in preparation.

R. Rosner, W. H. Tucker, and G. S. Vaiana, 1978 Ap. J., 2200, 643.

R. Rosner, L. Golub, and G. S. Vaiana, 1983, CfA Preprint 1719.

J. H. M. M. Schmitt, L. Golub, F. R. Harnden Jr., C. W. Maxson, R. Rosner., and G. S. Vaiana, 1985, Ap. J, 290, 307.

J. H. M. M. Schmitt, and R. Rosner, 1983, Ap. J., 265, 901.

S. Serio, G. Peres, G. S. Vaiana, L. Golub, R. Rosner, 1981, Ap. J., 243, 288.

S. Serio, 1983, Adv. Space Research, 2, 271.

S. Serio, 1985, Proc. ESA Workshop on a Cosmic Spoctroscopy Miseion, ESA SP-239, 59.

R. A. Stern, M. C. Zolcinski, S. K. Antiochos, and J. H. Underwood, 1981, Ap. J., 249, 647.

R. A. Stern, S. K. Antiochos; and F. R. Harnden Jr., 1986, Ap. J., 305, 417.

X. T. Strong, 1986, Proc. of the COSPAR Symposium on Symopsis of the Solar Maximum Arolysis (Toulouse), in press.

Y. Uchida, 1986, Proc. of the COSPAR Symposium on Stellar and Solar Activity (Toulouse), in press.

G. S. Vaiana, and R. Rosner, 1978, Ann. Rev. Astron. Ap., 16, 393.

G. S. Vaiana, S. Sciortino, 1987, this volume.

J. F. Yesecky, S. K. Antiochos, and J. H. Underwood, 1979, Ap. J, 233, 987.

M. C. Zolcinski, S. K. Antiochos, A. B. C. Walter, and R. A. Stern, 1982, Ap. J., 258, 177. 